Experimental Study of Turbulent Mixing in the Richtmyer-Meshkov Instability

C. R. Weber, N. S. Haehn, J. Oakley, D.

Rothamer, R. Bonazza

April 9, 2013

International Symposium on Shock Waves

Madison, WI, United States

July 14, 2013 through July 19, 2013 
This document was prepared as an account of work sponsored by an agency of the United States government. Neither the United States government nor Lawrence Livermore National Security, LLC, nor any of their employees makes any warranty, expressed or implied, or assumes any legal liability or responsibility for the accuracy, completeness, or usefulness of any information, apparatus, product, or process disclosed, or represents that its use would not infringe privately owned rights. Reference herein to any specific commercial product, process, or service by trade name, trademark, manufacturer, or otherwise does not necessarily constitute or imply its endorsement, recommendation, or favoring by the United States government or Lawrence Livermore National Security, LLC. The views and opinions of authors expressed herein do not necessarily state or reflect those of the United States government or Lawrence Livermore National Security, LLC, and shall not be used for advertising or product endorsement purposes. 


\title{
Experimental Study of Turbulent Mixing in the Richtmyer-Meshkov Instability
}

\author{
C. Weber ${ }^{\star}$, N. Haehnn ${ }^{\star \star}$, J. Oakley, D. Rothamer, R. Bonazza
}

\begin{abstract}
The Richtmyer-Meshkov instability (RMI) is experimentally investigated in a vertical shock tube using a broadband initial condition imposed on an interface between a helium-acetone mixture and argon $(A=0.7)$. The interface is created without the use of a membrane using a novel shear-layer technique, producing a statisticallyrepeatable, broadband initial condition to the RMI. The interface is accelerated by either a $M=1.6$ or $M=2.2$ planar shock wave, and the development of the ensuing mixing layer is investigated using planar laser-induced fluorescence (PLIF). The images suggest a transition to turbulent mixing occurring, characterized by the generation of smaller and chaotic features in the PLIF images, a homogenization of fluid, and the development of a $k^{-5 / 3}$ inertial range in the scalar energy spectra.
\end{abstract}

\section{Introduction}

An interface that separates two fluids of different densities is unstable to acceleration by a shock wave. In this interaction, called the Richtmyer-Meshkov instability (RMI) [1, 2], vorticity is baroclinically deposited on interfacial perturbations. The vorticity drives the growth of perturbation amplitudes and can lead to turbulent mixing between the two fluids. This instability is relevant to inertial confinement fusion, where mixing between different layers of the capsule can degrade performance by cooling the central hot-spot or diluting the fuel with ablator material.

The present set of experiments uses a novel initial condition setup, where a shear layer between two gases produces a broadband, membrane-free interface layer. The

University of Wisconsin-Madison, 1500 Engineering Dr., Madison, WI, 53706, USA

${ }^{\star}$ Present address: Lawrence Livermore National Laboratory, Livermore, CA

${ }^{\star}$ Present address: Intel Corporation, Phoenix, AZ 
post-shock development of this layer is studied using planar laser-induced fluorescence (PLIF) to obtain a description of the mixing characteristics of the turbulent RMI.

\section{Experimental Setup}

The present experiments were performed at the Wisconsin Shock Tube Laboratory. The shock tube is $9.1 \mathrm{~m}$ tall and has a $25.4 \times 25.4 \mathrm{~cm}^{2}$ internal cross section. These experiments use a gas interface with a mixture of helium and acetone vapor $(6.0 \pm 0.8 \%$ by volume) above and pure argon below, giving an Atwood number of 0.7. The flow of helium-acetone mixture is split, routing a portion to the top of the shock tube and the other portion to the interface section. First, an initially flat interface is formed by flowing the helium-acetone mixture into the top of the shock tube and argon into the bottom. Excess gas is evacuated through slots in the shock tube wall at the interface location. These slots are connected to a pair of vacuum pumps, ensuring a rapid outflow of gas. This method to create a flat, membraneless interface is similar to that developed for the University of Arizona shock tube [3] and used previously at the University of Wisconsin [4].

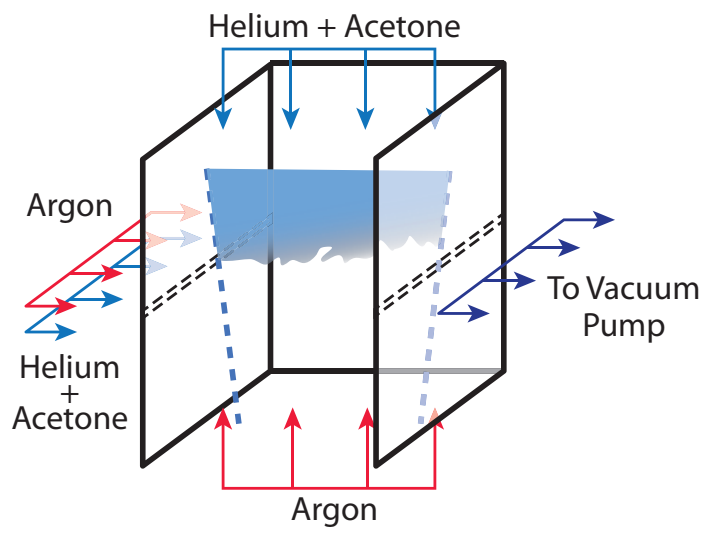

Fig. 1 Diagram of the interface location showing gas flowing from the top and bottom of the shock tube and from the left set of slots. The right set of slots are connected to a vacuum pump, removing excess gas. The planar laser sheet is shown as dashed lines and are illuminating the acetone vapor in the top gas.

The flat interface is perturbed by injecting the pure argon and the helium-acetone mixture horizontally through separate slots above and below the stagnation plane, respectively, while maintaining the vertical flow started previously. This flow configuration, shown in Fig. 1, was experimentally determined to provide the best initial condition in terms of scale content and repeatability. Perturbations form due to the 
buoyant interaction between the two streams and from the shear stress between this mixed layer and the pure argon. The superposition of the horizontal and vertical flows creates a continual flow towards the interface, ensuring that all the mixed gas is removed and the mixing layer remains statistically steady in time.

Two excimer lasers (Lambda Physik LPX 210i, 308 nm, 470 mJ/pulse, 28 ns pulse) are used for PLIF diagnostics. During each experiment, ten pre-shock images are recorded prior to the arrival of the shock wave to obtain a statistical description of the initial condition. To allow the laser to recharge and account for variability in experimental timing, the last recorded initial condition occurs $150-200 \mathrm{~ms}$ prior to the shock arriving at the interface. A pressure transducer above the interface is used to trigger the two lasers for two post-shock images based on the arrival of the shock wave. The images are all recorded using three thermoelectrically cooled (to $-60^{\circ} \mathrm{C}$ ) Andor CCD cameras (model DV434-BU2).
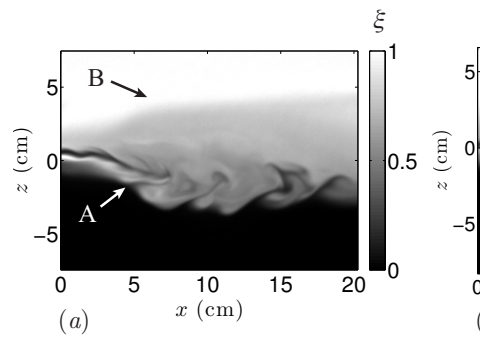
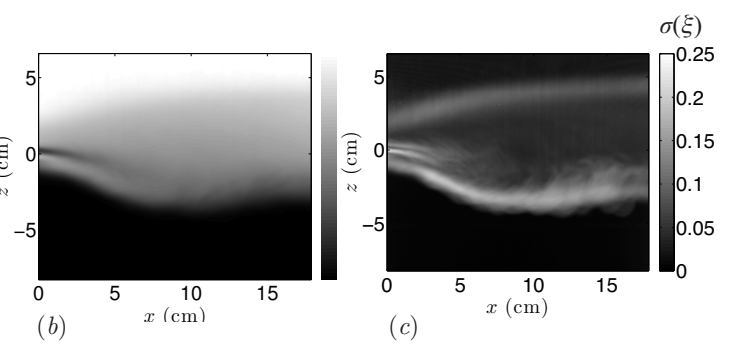

(c)

Fig. 2 Initial condition images. (a) A sample initial condition image, processed so intensity corresponds to light-gas mole fraction. $(b)$ Mole fraction ensemble average and $(c)$ standard deviation from 100 images

Figure 2(a) shows a sample initial condition image, corrected so that the signal intensity corresponds to relative acetone concentration, which is also directly proportional to the light-gas mole fraction, $\xi$. In the image, the gases are injected from the left near $z=0 \mathrm{~cm}$. The injected stream of pure argon is visible as a dark horizontal band at $z \approx 0.5 \mathrm{~cm}$. The injected helium-acetone mixture is visible below the argon stream. Approximately $5 \mathrm{~cm}$ to the right right of the injection location (feature A), the two gas streams begin mixing and the individual streams are no longer apparent. Perturbations develop on the lower edge of this mixing region due to the velocity difference of the mixture stream and the ambient argon. The boundary between the mixed gas entering from the left and the helium-acetone mixture entering from the top of the shock tube is identified as feature B. The gradient at the top of the mixing region is diffuse and lacks noticeable perturbations. Between the top contour (feature B) and the bottom shear surface (feature A), the average mole fraction is $\xi \approx 0.6$. The ensemble average and standard deviation from 100 images are shown in Figs. 2(b) and (c). The most significant temporal fluctuations occur at the bottom shear surface, where the light-gas mole fraction has a standard deviation of 0.2. This initial condition is characterized further in Weber et al. [5]. 


\section{Post-Shock Results and Analysis}
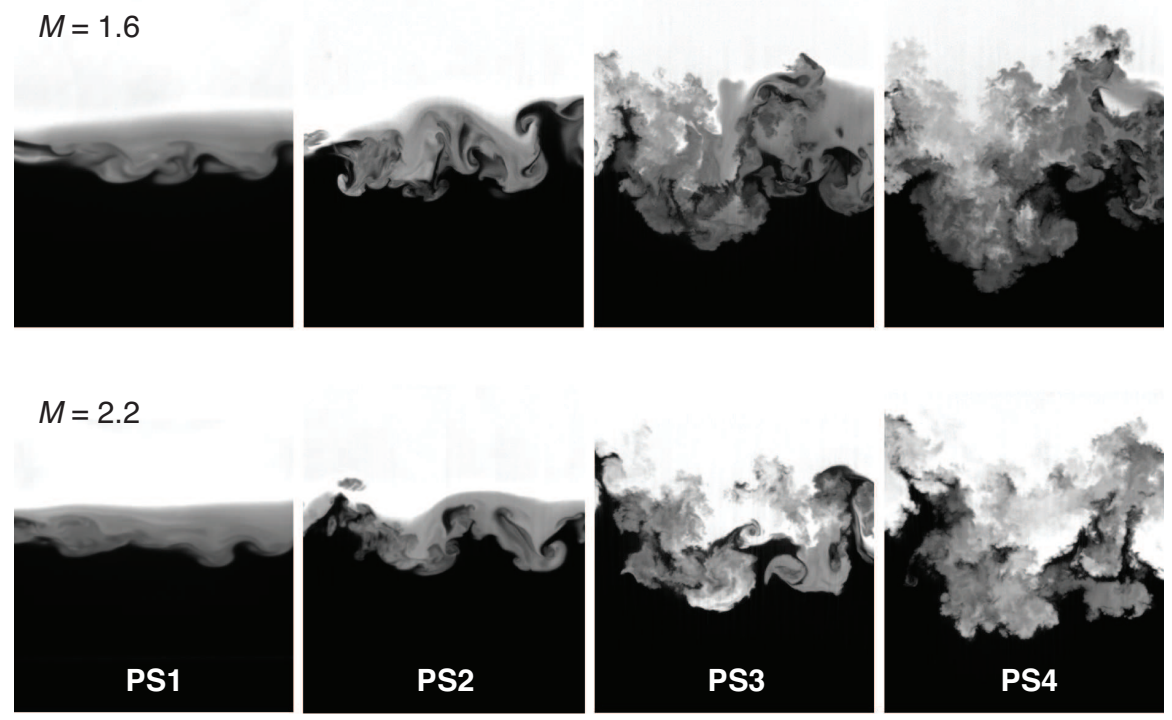

Fig. 3 Selected images from $M=1.6$ (top) and $M=2.2$ (bottom) experimental sequence. The width of each image is $14.0 \mathrm{~cm}$.

The interface is accelerated by an incident shock wave of strength $M=1.57 \pm$ 0.02 or $M=2.23 \pm 0.02$. At each Mach number, images from four post-shock times are obtained (termed: PS1-PS4). Figure 3 shows a sample of corrected PLIF images from the $M=1.6$ (top) and $M=2.2$ (bottom) experiments. From left to right, the experimental times after the initial shock interaction are: $0.14 \mathrm{~ms}, 0.88 \mathrm{~ms}, 2.16 \mathrm{~ms}$, and $3.84 \mathrm{~ms}$ for the $M=1.57$ images and $0.10 \mathrm{~ms}, 0.44 \mathrm{~ms}, 1.12 \mathrm{~ms}$, and $2.05 \mathrm{~ms}$ for the $M=2.2$ images. The last two images in each row are from the same experiment, whereas all other images are from different experiments.

The images show that the large-scale extent of the mixing layer is growing, while the fluid within the layer is becoming more mixed and turbulent. The earliest postshock images seem to have features similar to those seen in the initial condition images, but the gradients are somewhat sharper, due to the compression from the shock wave. At the PS2 time, the layer is dominated by several spikes of heavy gas penetrating into the mixed gas. Coherent vortices are noticeable at this time and the interface contours appear relatively smooth. By the PS3 time, the smoothness that appeared along the interface is gone and many small-scale features are present. This trend continues into the PS4 time, where the mixing layer appears to be in a fully-turbulent state. Isolated regions in the PS3 images can be noticed where the 
layer remains relatively smooth; these no longer exist by the PS4 time, where the full layer appears engulfed by turbulence.

The similarities between the two Mach numbers are remarkable given the $\sim 2 \times$ difference in interface velocity. The post-shock image number (i.e. PS1, PS2, etc.) denotes the shock tube window used for the image and, between the two Mach numbers, represents the same post-shock travel distance. Thus the post-shock travel distance appears to qualitatively capture the turbulent evolution of the mixing layer. A few differences are apparent between the two Mach numbers. The greater compression of the $M=2.2$ flow results in a thinner mixing layer at the same window location. The composition of the layer also appears different at the last two times, but this difference is better seen in the probability density function (PDF) of the mole fraction.

The evolution of $\operatorname{PDF}(\xi)$ within the full mixing layer (within $0.05<\langle\xi\rangle<0.95$, where $\langle\xi\rangle$ denotes the spanwise averaged mole fraction) is shown in Fig. 4 for the (a) $M=1.6$ data and (b) $M=2.2$ data. Each curve represents an average over 2040 experiments. PS1 has a similar mole fraction distribution as the initial condition (not shown) due to its early post-shock time. Due to the shear layer initial condition technique, a local peak in the PDF exists near $\xi \approx 0.4-0.5$. This represents a reservoir of fluid that can mix with either the $\xi=0$ or 1 fluid. The PDFs show that this local peak reduces over time and appears to mix with the lighter $(\xi=1)$ fluid. This process occurs more rapidly in the $M=2.2$ case and results in an increase in the fluid near $\xi \sim 0.8$. This bias for mixing of the lighter fluids has been noticed elsewhere and is attributed to the greater inertia of the heavy fluid [6].
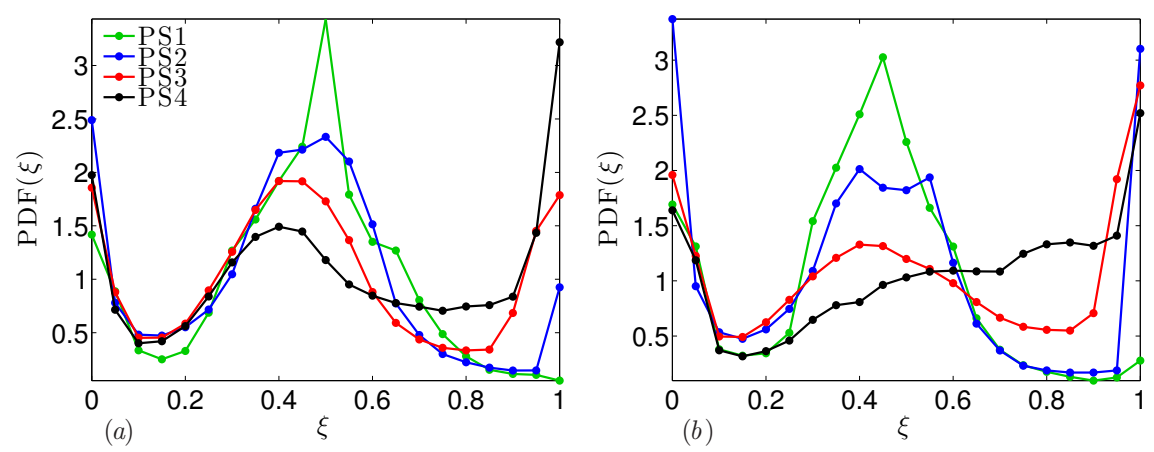

Fig. 4 PDF of mole fraction within $0.05<\langle\xi\rangle<0.95$, (a) $M=1.6$ and $(b) M=2.2$.

The scale distribution of the mole fraction fields is reported here using onedimensional scalar energy spectra. The spectra are computed horizontally within the region $0.1<\langle\xi\rangle<0.7$ and combined through an interlacing technique [7] to create a ensemble averaged spectrum for each time. Figure 5 shows the 1D energy spectra for the five times from the $(a) M=1.6$ and $(b) M=2.2$ data. The spectra from the IC and PS1 lie very close to each other, as would be expected given the 

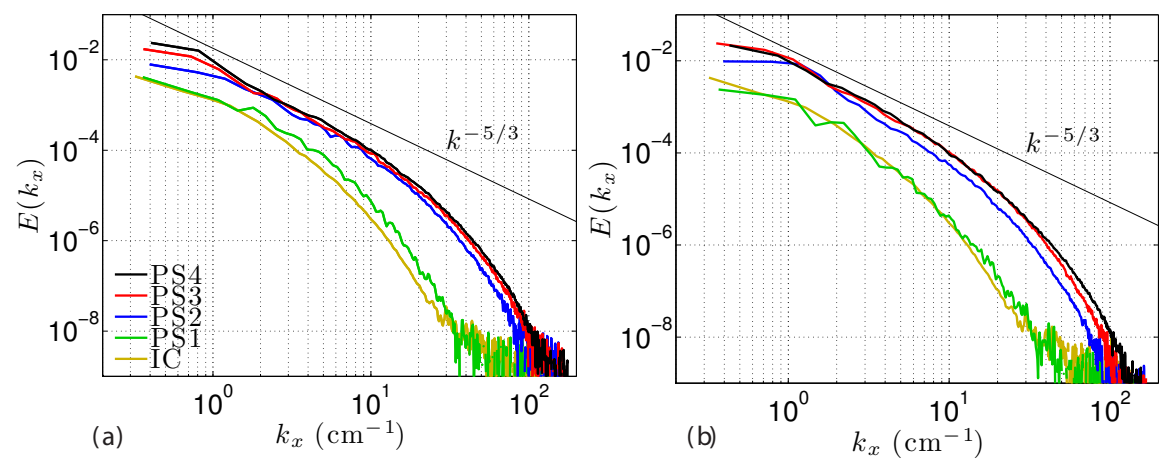

Fig. 5 (a) One-dimensional scalar variance energy spectra, (a) $M=1.6$ and (b) $M=2.2$.

very early time of PS1. Between PS1 and PS2 the magnitude of the spectrum increases, representing an increase in scalar variance. The spectra of last three times, PS2-PS4, are very similar, which is interesting given the visual difference between the corresponding images in Fig. 3. The magnitude of the high wavenumber region is increasing through the latest time. An apparent $k^{-5 / 3}$ inertial range is noticeable at the latest three times. This inertial range manifests for approximately a decade in wavenumbers before an exponential dissipation region is observed. In the $M=2.2$ data, the slope of the inertial range appears slightly steeper than $k^{-5 / 3}$.

\section{Conclusions}

These experiments present a unique initial condition for studying the RichtmyerMeshkov instability and the development of a turbulent mixing layer. In $M=1.6$ and $M=2.2$ experiments, turbulent mixing is observed at the latest times, characterized by the generation of smaller and chaotic features in the PLIF images, a homogenization of fluid, and the development of an inertial range $\left(\sim k^{-5 / 3}\right)$ in the scalar energy spectra. Between the two Mach numbers, the layer transitions to a turbulent state at a similar interface travel distance, which can provide insight into the mechanisms behind a time dependent transition to turbulence.

\section{Acknowledgements}

This work was performed under the auspices of the U.S. Department of Energy by Lawrence Livermore National Laboratory under Contract DE-AC52-07NA27344. Support to the University of Wisconsin was provided by U.S. DOE Grant No. DEFG52-06NA26196. 


\section{References}

1. R. D. Richtmyer. Taylor instability in shock acceleration of compressible fluids. Communications on Pure and Applied Mathematics, 13(2):297-319, 1960.

2. E. E. Meshkov. Instability of a shock wave accelerated interface between two gases. NASA Technical Translation, 13:1-14, 1970.

3. M. A Jones and J. W Jacobs. A membraneless experiment for the study of Richtmyer-Meshkov instability of a shock-accelerated gas interface. Physics of Fluids, 9(10):3078-3085, 1997.

4. B. Motl, J. Oakley, D. Ranjan, C. Weber, M. Anderson, and R. Bonazza. Experimental validation of a Richtmyer-Meshkov scaling law over large density ratio and shock strength ranges. Physics of Fluids, 21(12):126102, 2009.

5. C. Weber, N. Haehn, J. Oakley, D. Rothamer, and R. Bonazza. Turbulent mixing measurements in the Richtmyer-Meshkov instability. Physics of Fluids, 24(7):074105, 2012.

6. D. Livescu and J. R. Ristorcelli. Variable-density mixing in buoyancy-driven turbulence. Journal of Fluid Mechanics, 605:145-180, May 2008.

7. S. A Kaiser and J. H Frank. Imaging of dissipative structures in the near field of a turbulent non-premixed jet flame. Proceedings of the Combustion Institute, 31(1):1515-1523, 2007. 